\title{
Dietary Practices, Addictive Behavior and Bowel Habits and Risk of Early Onset Colorectal Cancer: a Case Control Study
}

\author{
Naveed Ali Khan', Mehwish Hussain ${ }^{2 *}$, Ata ur Rahman', Waqas Ahmed \\ Farooqui $^{2}$, Abdur Rasheed ${ }^{2}$, Amjad Siraj Memon ${ }^{1}$
}

\begin{abstract}
Background: The abrupt rise of colorectal cancer in developing countries is raising concern in healthcare settings. Studies on assessing relationships with modifiable and non-modifiable risk factors in the Pakistani population have been limited. The present investigation was designed to examine associations of dietary practices, addictive behavior and bowel habits in developing colorectal cancer (CRC) among patients in a low-resource setup. Materials and Methods: An age-gender matched case control study was conducted from October 2011 to July 2015 in Karachi, Pakistan. Cases were from the surgical oncology department of a public sector tertiary care hospital, while their two pair-matched controls were recruited from the general population. A structured questionnaire was used which included questions related to demographic characteristics, family history, dietary patterns, addictive behavior and bowel habits. Results: A family history of cancer was associated with a 2.2 fold higher chance of developing CRC. Weight loss reduced the likelihood 7.6 times. Refraining from a high fat diet and consuming more vegetables showed protective effects for CRC. The risk of CRC was more than twice among smokers and those who consumed Asian specific addictive products as compared to those who avoid using these addictions (ORsmoking: 2.12, 95\% CI: 1.08 - 4.17, ORpan: 2.92, 95\% CI: 1.6 - 5.33, ORgutka: 2.13, 95\% CI: 1.14 - 3.97). Use of NSAID attenuated risk of CRC up to 86\% (OR: 0.14, 95\% CI: 0.07 - 0.31 ). Conclusions: Most of the findings showed concordance with the literature elucidating protective effects of consuming vegetables and low fat diet while documenting adverse associations with family history, weight loss, constipation and hematochezia. Moreover, this study highlighted Asian specific indigenous addictive products as important factors. Further studies are needed to validate the findings produced by this research.
\end{abstract}

Keywords: Colorectal cancer - dietary practices - addictive behaviors - bowel habits - risk factors - Pakistan

Asian Pac J Cancer Prev, 16 (17), 7967-7973

\section{Introduction}

Colorectal cancer (CRC) is the second leading cause of death due to cancer globally in both men and women (Torre et al., 2015). Once known as disease of industrial and developed countries, CRC extended its pace steadily in developing countries (Bishehsari et al., 2014; Torre et al., 2015). Over the past decade the disease rate is uprising many folds higher in Middle-East and different countries of Asia (Bishehsari et al., 2014; Malekzadeh et al., 2015).

The recent increasing trend in incidence of CRC is mainly attributed to the shift in lifestyle factors. Lack of physical activity, western dietary pattern and increased use of tobacco are the most reported risk factors for the CRC (Mehta and Shike, 2014). High body mass index (Lund et al., 2011; Mehta and Shike, 2014; Simons et al., 2015), abundant use of red and processed meat (Vargas and Thompson, 2012; Aykan et al., 2015) and high fat diet (Bishehsari et al., 2014) are also strongly linked with the development of CRC. Literature also reported considerable relationship of alcohol and tobacco use products (Bishehsari et al., 2014; Torre et al., 2015) with substantial concerns on cigarette smoking (Gong et al., 2012). According to recent update by World Cancer Research Fund (WCRF)/American Institute of Cancer Research (AICR), alcohol intake increased $10 \%$ risk of CRC (Roswall and Weiderpass, 2015). Genetic variation ubiquitously predisposed its deteriorative effect worldwide (Vargas and Thompson, 2012; Bishehsari et al., 2014; Álvarez-Cuenllas et al., 2015; Simons et al., 2015).

Different aetiological studies from various regions of the world have reported varying estimates of risk when it comes to lifestyle and dietary factors and their role in development of CRC. The underlying explanation might be the differences in consumption of certain products, their processing and cooking practices. Low consumption of vegetables and high consumption of red meat were the most frequent risk factors among CRC patients from Turkey (Aykan et al., 2015). A study from Egypt reported higher odds of red meat consumption followed 
by preserved food and artificial sweeteners (Mahfouz et al., 2014). Results from a Canadian population based study detected ominous effect of meat and sugary diets and protective effect of plant based diet (Chen et al., 2015).

Literature on identifying risk factors of CRC from South-Asian regions is limited in several dimensions. Most studies conducted in our region have looked at the epidemiological profile of patients who are presenting at hospital (Anwar et al., 2008; Bhurgri et al., 2011) along with their short term and long term outcomes following treatment (Khan et al., 2011a; Khan et al., 2011b; Malik et al., 2015). However, there is limited evidence on the role of lifestyle and indigenous dietary factors and practices with the development of CRC. To our knowledge no study was found elucidating factors leading to development of $\mathrm{CRC}$ in our region. We therefore perceived that there is need of generating evidence on the risk factors of CRC in our population. The focus of this research was to assess risk factors for CRC among less resourced Pakistani population.

\section{Materials and Methods}

\section{Study Setting}

This case control study was carried out during October 2011 to July 2015 in Karachi, Pakistan. Participants were recruited from surgical oncology units of Civil Hospital Karachi (CHK). CHK is 1,900 bedded tertiary care hospital with yearly enrollment is approximately over three thousand patients. The annual flow of cancer patients in surgical oncology unit is approximately $5 \%$ of all cancer. The CHK is affiliated with Dow University of Health Sciences (DUHS) which is a prominent public sector institute in Pakistan.

\section{Data collection method}

Cases: The clinical examination of the cases was performed by a trained specialist surgeon of all patients on whom there was a suspicion of CRC. All these patients with suspected CRC during the study period were selected as cases. The CRC diagnosis was then confirmed by the histopathology examination of the biopsy specimen. The classification of patients for different stages of CRC was done using Duke's staging system (NICE, 2011).

\section{Controls}

For each case, two age and gender matched controls were invited to participate in the study. One group of control was sibling while another group was neighbor of the patient. In case of absence of any counterpart sibling or neighbor, a friend of the patient with matched age and gender residing in similar socio-economic area as of patient was recruited in the study. This method of selection was kept to maintain equilibrium in socio-economic status between cases and controls of the study. Data from control was collected by trained interviewers. The training to the data collectors was given by investigators of the study.

\section{Ethical Consideration}

Permission was obtained by the Departmental Research Review committee for collecting data from the oncology departments. An informed consent was obtained from the participants bearing age 18-65 years. Consent for participants less than 18 years was taken from their parent while consent for the participants more than 65 years of age was taken from presenting surrogate (usually son or daughter of the elderly). Participants were explicitly informed about the purpose of the study. Confidentiality and autonomy of the participants were pledged to them prior to data collection.

\section{Research Instrument}

We used a structured questionnaire which was divided into six major parts including demographic characteristics, family history, dietary practices, addictive behaviors, bowel habits and drug history of participants. Clinical examination and duke's staging system were additional sections while collecting data from cases. The description of variables in these sections is mentioned below.

\section{Demographic characteristics}

Other than age and gender, ethnicity, socio-economic status and residential area were noted down for each participant.

\section{Family history}

Presence of any type of cancer in the family of the cases and controls was noted down. History of bleeding per rectum in the family was also investigated. In case of presence of any of these two histories, relationship of the diseased with participant was additionally asked.

Weight loss and loss of appetite were also examined for all participants during study period.

\section{Dietary practices}

In dietary practices consumption of rice and rice powder was asked. Also, whether a subject was vegetarian, non-vegetarian, used refine foods or high fat diets. A subject was referred as vegetarian who refrained eating any kind of meat from animals, fish or fowl, notwithstanding subsists oneself on vegetables, fruits, grains etc. Non-vegetarian, on the other hand, were alluded as patients who abstained themselves from any food derived from vegetables. Consumption of refined grains such as corn flakes, pastas, macaroni, noodles, pizza and refined sugars such as cakes, cookies, desserts, fruit drinks were classified as refined foods. High fat diets were considered if patient consumed butter, cheese, whole eggs, nuts, yogurt etc. continually.

\section{Addictive behaviors}

Other than habits of smoking, alcohol drinking and drug intake, participants were asked about eating practices of indigenous Asian specific addictive products such as chhaliah (areca nut), paan (betel quid), gutka and naswar (snuff). Chhaliah is an ancient cultural and widely consumed pharmacologic staple. It is commonly chewed with other indigenous Asian specific addictive products such as paan and gutka especially in the subcontinent (India, Pakistan and Bangladesh) (Hooper et al., 2015). This psychoactive food agent has established sufficient evidences in producing carcinogenicity in 
Dietary Practices, Addictive Behavior and Bowel Habits and Risk of Early Onset Colorectal Cancer: a Case Control Study

mouth and esophagus (Garg et al., 2014). Researchers have proclaimed its harmful effect also on reproductive organs and gastrointestinal tracks.

Paan used with and without tobacco has shown positive association with oral carcinoma. Recent literature from Pakistan revealed that individuals who eat paan with tobacco had twice the risk of cancer than those who eat it without tobacco (Merchant and Pitiphat, 2015).

Gutka is another recent addictive product which contains ingredients of sun-fried finely chopped tobacco, areca nut, catechu, slaked lime, sweeteners and flavorings. Gutka is also set responsible for the development of oral and pancreatic cancer along with adverse reproductive complications in female (Banerjee et al., 2014).

Naswar is mostly used by natives from north-west frontier region of Pakistan. Its ingredients contain powdered tobacco, slaked lime and indigo. This niche smokeless product is traditionally consumed by dipping, a method of placing a pinch of naswar under the tongue and stored for a long period (Basharat et al., 2012). The toxin effect of its ingredients demonstrated leading cause in the development of oral and oesophageal cancer (Saeed et al., 2012).

\section{Bowel habits}

Seven parameters were observed for assessing abnormalities in bowel habits which were referred as plausible factors for CRC. First was suffering abdominal distention which was described as bloating of stomach and waist or increased pressure in abdomen, pain and/ or cramping. Second was constipation as obstructive defecation, third was diarrhea as defined by WHO as "passage of three or more loose or liquid stools per day" and fourth irritable bowel syndrome that is mixed symptoms of constipation, diarrhea and abdominal distension. Investigations were also made up for frequent bowel movements in whole day or in morning specifically, rectal tenesmus which was defined as feeling of difficulty or inability of evacuating complete bowel during defecation and hematochezia defined as passage of blood through rectum, usually with or in feces.

\section{Drug history}

For cases, history of non-steroidal anti-inflammatory drugs (NSAID), adjuvant, neo-adjuvant treatment or any other treatment recommended by oncologist was noted down. Controls were asked if they had ever used any antibiotics in last 12 months.

\section{Statistical analysis}

All the categorical variables under study were described with frequency and percentages. Age when taken as continuous variables was presented as mean \pm SD. At univariable analysis stage, chi-square test was run to check association of CRC with family history, dietary practices, bowel habits, addictive behaviors and drug history. Bivariate logistic regression model was executed to obtain crude odds ratio assessing likelihood of CRC due to individual factor under study. At this stage, factors exhibiting $\mathrm{p}$ value less than or equal to 0.25 were included in multivariable analyses. Results were reported as odds ratio with $95 \%$ confidence interval. The value of 0.05 was set as threshold for showing significant association between CRC status and factors under study.

\section{Results}

\section{Demographic characteristics}

A total of 74 CRC patients participated in the study. Of them $39(52.7 \%)$ were female and $35(47.3 \%)$ were male. The ratio of cases to controls was 1:2 (Table 1). The average age of cases was $41.47 \pm 15.48$ years while controls were $41.47 \pm 15.54$ years of age $(\mathrm{P}>0.999)$. More than half participants belonged to low socio-economic status.

\section{Family history}

Presence of cancer in the family was reported by $12.6 \%$ of the participants. CRC was 2.2 folds higher among participants with family history of cancer $(\mathrm{OR}=2.23$ 95\% CI: 1.0-4.98, $\mathrm{P}=0.045$ ). Weight loss was observed by 106 while loss of appetite by 93 individuals. The risk of CRC among individuals reporting loss of appetite was 4.63 times greater than those who did not observe the

Table 1. Association of Personal History with Colorectal Cancer

\begin{tabular}{|c|c|c|c|c|c|}
\hline \multirow{2}{*}{\multicolumn{2}{|c|}{ Characteristics }} & \multicolumn{2}{|c|}{ Subject Type } & \multirow{2}{*}{$\begin{array}{c}\text { Univariable Analysis } \\
\text { ORa }(95 \% \mathrm{CI})\end{array}$} & \multirow{2}{*}{$\begin{array}{c}\text { Multivariable Analysis } \\
\text { ORb }(95 \% \text { CI })\end{array}$} \\
\hline & & Control $(n=148)$ & Cases $(n=74)$ & & \\
\hline \multicolumn{6}{|l|}{ Demographic Factors } \\
\hline \multirow[t]{2}{*}{ Gender } & Male & $70(47.3 \%)$ & $35(47.3 \%)$ & 1 & 1 \\
\hline & male & $78(52.7 \%)$ & $39(52.7 \%)$ & $1.00(0.57-1.75)$ & $0.46(0.10-2.09)$ \\
\hline \multirow[t]{3}{*}{ Age in years } & 5.47) & $41.47 \pm 15.48$ & $41.47 \pm 15.54$ & & \\
\hline & o 24) & $22(14.9 \%)$ & $11(14.9 \%)$ & 1 & 1 \\
\hline & 5-85) & $126(85.1 \%)$ & $63(85.1 \%)$ & $1.00(0.46-2.19)$ & $1.76(0.46-6.75)$ \\
\hline \multicolumn{6}{|l|}{ Personal History } \\
\hline \multirow[t]{2}{*}{ Family History of Cancer } & No & $134(90.5 \%)$ & $60(81.1 \%)$ & 1 & 1 \\
\hline & Yes & $14(09.5 \%)$ & $14(18.9 \%)$ & $2.23(1.00-4.98)^{*}$ & $2.97(0.64-13.84)$ \\
\hline \multirow[t]{2}{*}{ Weight Loss } & No & $100(67.6 \%)$ & $16(21.6 \%)$ & 1 & 1 \\
\hline & Yes & $48(32.4 \%)$ & $58(78.4 \%)$ & $7.55(3.94-14.49)^{* *}$ & $5.92(1.68-20.88)$ \\
\hline \multirow[t]{2}{*}{ Loss of Appetite } & No & $104(70.3 \%)$ & $25(33.8 \%)$ & 1 & 1 \\
\hline & Yes & $44(29.7 \%)$ & $49(66.2 \%)$ & $4.63(2.55-8.42) * *$ & $1.97(0.61-6.41)$ \\
\hline
\end{tabular}

$\mathrm{ORa}=$ unadjusted odds ratios; $\mathrm{ORb}=$ odds ratios adjusted for all independent variables, $\mathrm{CI}=$ confidence intervals; **Significant at $1 \% ; *$ Significant at $5 \%$ 
Naveed Ali Khan et al

same. Weight loss mounted the risk almost twice the risk due to loss of appetite (OR=7.55, 95\% CI: 3.94-14.49, $\mathrm{P}<0.0001)$.

\section{Dietary practices}

Rice and rice powder were used by $139(62.7 \%)$ participants (Table 2). Each 6 out of 10 respondents were vegetarian whereas less than a quarter were classified as non-vegetarian. Half of the sampled individuals used refined foods. High fats diets were consumed by 101
(45.5\%) participants. All the dietary practices showed protective effect for CRC except for high fat diet. Individuals consuming high fats diet had $98 \%$ higher chances to endure CRC as compare to those who avoid using such diets $(\mathrm{OR}=1.98,95 \% \mathrm{CI}$ : 1.13-3.49, $\mathrm{P}=0.017)$.

\section{Addictive behaviors}

The habit of smoking was observed among 159 participants. The proportion of this habit was significantly larger in cases as compare to their control counterparts.

Table 2. Association of Dietary Practices, Addictive Behaviors with Colorectal Cancer

\begin{tabular}{|c|c|c|c|c|c|}
\hline \multirow{2}{*}{ Characteristics } & & \multicolumn{2}{|c|}{ Subject Type } & \multirow{2}{*}{$\begin{array}{l}\text { Univariable Analysis } \\
\text { OR }^{\mathrm{a}}(95 \% \mathrm{CI})\end{array}$} & \multirow{2}{*}{$\begin{array}{c}\text { Multivariable Analysis } \\
\operatorname{OR}^{\mathrm{b}}(95 \% \mathrm{CI})\end{array}$} \\
\hline & & Control $(n=148)$ & Cases $(n=74)$ & & \\
\hline \multicolumn{6}{|l|}{ Dietary Practices } \\
\hline \multirow[t]{2}{*}{ Rice and Powder } & No & $39(26.4 \%)$ & $44(59.5 \%)$ & 1 & 1 \\
\hline & Yes & $109(73.6 \%)$ & $30(40.5 \%)$ & $0.24(0.14-0.44)^{* *}$ & $0.31(0.10-1.00)$ \\
\hline \multirow{2}{*}{ Vegetarian } & No & $27(18.2 \%)$ & $50(67.6 \%)$ & 1 & 1 \\
\hline & Yes & $121(81.8 \%)$ & $24(32.4 \%)$ & $0.11(0.06-0.20)^{* *}$ & $0.06(0.02-0.22)$ \\
\hline \multirow[t]{2}{*}{ Non Vegetarian } & No & $114(77 \%)$ & $58(78.4 \%)$ & 1 & \\
\hline & Yes & $34(23 \%)$ & $16(21.6 \%)$ & $0.93(0.47-1.81)$ & \\
\hline \multirow[t]{2}{*}{ Refined Foods } & No & $38(25.7 \%)$ & $73(98.6 \%)$ & 1 & \\
\hline & Yes & $110(74.3 \%)$ & $1(01.4 \%)$ & $0.01(0.00-0.04)^{* *}$ & \\
\hline \multirow[t]{2}{*}{ High Fat Diet } & No & $89(60.1 \%)$ & $32(43.2 \%)$ & 1 & 1 \\
\hline & Yes & $59(39.9 \%)$ & $42(56.8 \%)$ & $1.98(1.13-3.49)^{*}$ & $1.64(0.58-4.62)$ \\
\hline \multicolumn{6}{|l|}{ Addictive Behaviors } \\
\hline \multirow[t]{2}{*}{ Smoking } & No & $49(33.1 \%)$ & $14(18.9 \%)$ & 1 & 1 \\
\hline & Yes & $99(66.9 \%)$ & $60(81.1 \%)$ & $2.12(1.08-4.17)^{*}$ & $1.27(0.24-6.85)$ \\
\hline \multirow[t]{2}{*}{ Pan } & No & $116(78.4 \%)$ & $41(55.4 \%)$ & 1 & 1 \\
\hline & Yes & $32(21.6 \%)$ & $33(44.6 \%)$ & $2.92(1.60-5.33) * *$ & $1.34(0.23-7.69)$ \\
\hline \multirow[t]{2}{*}{ Gutka } & No & $118(79.7 \%)$ & $48(64.9 \%)$ & 1 & 1 \\
\hline & Yes & $30(20.3 \%)$ & $26(35.1 \%)$ & $2.13(1.14-3.97)^{*}$ & $2.61(0.64-10.64)$ \\
\hline \multirow{2}{*}{ Niswar/Chalia } & No & $134(90.5 \%)$ & $56(75.7 \%)$ & 1 & 1 \\
\hline & Yes & $14(09.5 \%)$ & $18(24.3 \%)$ & $3.08(1.43-6.61)^{* *}$ & $0.75(0.13-4.30)$ \\
\hline \multirow[t]{2}{*}{ Alcohol/Drug } & No & $128(86.5 \%)$ & $46(62.2 \%)$ & 1 & 1 \\
\hline & Yes & $20(13.5 \%)$ & $28(37.8 \%)$ & $3.90(2.00-7.58) * *$ & $2.97(0.35-24.93)$ \\
\hline \multirow[t]{2}{*}{ Any one of the above } & Not Addicted & $24(16.2 \%)$ & $2(2.7 \%)$ & 1 & \\
\hline & Addicted & $124(83.8 \%)$ & $72(97.3 \%)$ & $6.97(1.60-30.35)^{* *}$ & \\
\hline
\end{tabular}

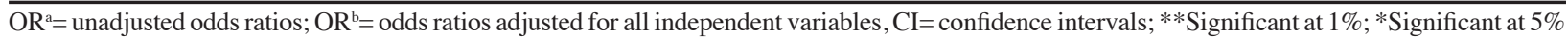

Table 3. Association of Bowel Habits with Colorectal Cancer

\begin{tabular}{|c|c|c|c|c|c|}
\hline \multirow[t]{2}{*}{ Characteristics } & & \multicolumn{2}{|c|}{ Subject Type } & \multirow{2}{*}{$\begin{array}{c}\text { Univariable Analysis } \\
\text { ORa }(95 \% \mathrm{CI})\end{array}$} & \multirow{2}{*}{$\begin{array}{c}\text { Multivariable Analysis } \\
\text { ORb }(95 \% \text { CI })\end{array}$} \\
\hline & & Control $(n=148)$ & Cases $(n=74)$ & & \\
\hline \multicolumn{6}{|l|}{ Bowel Habits } \\
\hline \multirow[t]{2}{*}{ Abdominal Distention } & No & $95(64.2 \%)$ & $52(70.3 \%)$ & 1 & \\
\hline & Yes & $53(35.8 \%)$ & $22(29.7 \%)$ & $0.76(0.42-1.38)$ & \\
\hline \multirow[t]{2}{*}{ Constipation } & No & $100(67.6 \%)$ & $33(44.6 \%)$ & 1 & 1 \\
\hline & Yes & $48(32.4 \%)$ & $41(55.4 \%)$ & $2.59(1.46-4.59)^{* *}$ & $1.03(0.34-3.13)$ \\
\hline \multirow[t]{2}{*}{ Diarrhea } & No & $121(81.8 \%)$ & $57(77 \%)$ & 1 & \\
\hline & Yes & $27(18.2 \%)$ & $17(23 \%)$ & $1.34(0.68-2.65)$ & \\
\hline \multicolumn{6}{|l|}{ Irritable bowel syndrome } \\
\hline & No & $135(91.2 \%)$ & $65(87.8 \%)$ & 1 & \\
\hline & Yes & $13(08.8 \%)$ & $9(12.2 \%)$ & $1.44(0.59-3.54)$ & \\
\hline \multirow[t]{2}{*}{ Increased frequency } & No & $128(86.5 \%)$ & $60(81.1 \%)$ & 1 & \\
\hline & Yes & $20(13.5 \%)$ & $14(18.9 \%)$ & $1.49(0.71-3.16)$ & \\
\hline \multirow[t]{2}{*}{ Increased frequency in morning } & No & $132(89.2 \%)$ & $54(73 \%)$ & 1 & 1 \\
\hline & Yes & $16(10.8 \%)$ & $20(27 \%)$ & $3.06(1.47-6.34)^{* *}$ & $0.62(0.15-2.63)$ \\
\hline \multirow[t]{2}{*}{ Hematochezia } & No & $130(87.8 \%)$ & $30(40.5 \%)$ & 1 & 1 \\
\hline & Yes & $18(12.2 \%)$ & $44(59.5 \%)$ & $10.59(5.38-20.85)^{* *}$ & $13.6(3.95-46.76)$ \\
\hline \multirow[t]{2}{*}{ Use of NSAID } & No & $75(50.7 \%)$ & $65(87.8 \%)$ & 1 & 1 \\
\hline & Yes & $73(49.3 \%)$ & $9(12.2 \%)$ & $0.14(0.07-0.31)^{* *}$ & $0.11(0.03-0.41)$ \\
\hline
\end{tabular}

$\mathrm{ORa}=$ unadjusted odds ratios; ORb $=$ odds ratios adjusted for all independent variables, $\mathrm{CI}=$ confidence intervals; $* *$ Significant at $1 \%$; Significant at $5 \%$ 
Dietary Practices, Addictive Behavior and Bowel Habits and Risk of Early Onset Colorectal Cancer: a Case Control Study

Smoking evoked the likelihood of CRC 2.1 folds more as compare to non-smokers healthy individuals $(\mathrm{OR}=2.12$, 95\% CI: $1.08-4.17, \mathrm{P}=0.027)$. Chhaliah and naswar chewed by 32 participants only but showed 3.1 times significantly higher chances for the development of CRC. Paan was consumed by nearly $30 \%(n=65)$ individuals. Consumption of paan increased the risk of CRC up to 2.9 times more than those who did not use paan $(\mathrm{OR}=2.92$, 95\% CI: $1.6-5.33, \mathrm{P}<0.001)$. Nearly one quarter of respondents used gutka and imperiled the likelihood 2.1 times more for the development of $\mathrm{CRC}(\mathrm{OR}=2.1395 \%$ CI: 1.14-6.61, $\mathrm{P}=0.016)$. Intake of alcohol and drugs elevated the risk of CRC to 3.9 times $(\mathrm{P}<0.001)$. Overall, $88 \%$ of the participants used any one of these addictions. Among cases this proportion was $97.3 \%$. The risk of CRC among those who were took any of the above addictive food was 7 folds higher as compare to those who refrained using any addictive food $(\mathrm{P}=0.003)$.

\section{Bowel Habits}

Abdominal distention was observed by each 1 out of 3 participants (Table 3 ). However, it was not significantly associated with $\mathrm{CRC}(\mathrm{P}=0.37)$. Constipation was notified by $40 \%$ of the sampled individuals and the likelihood of CRC was 2.6 folds higher among them $(\mathrm{OR}=2.59,95 \%$ CI: $1.46-4.59, \mathrm{P}<0.001)$. Diarrhea was reported by 44 participants while irritable bowel syndrome by only 22 of sampled individuals under study. None of these bowel habits showed significant association in the development of CRC. Increase frequencies of bowel movement in day time or morning time specifically were also insignificantly associated with the disease under study. Only $8.1 \%$ $(n=18)$ participants abided rectal tenesmus and all of them were cases. None of the control experienced this bowel complications $(\mathrm{P}=0.001)$. A strong relationship was determined between hematochezia and CRC in our study. Hematochezia induced 10.6 times higher chances for developing CRC (OR=10.59, 95\% CI: 5.38-20.85, $\mathrm{P}<0.0001)$.

\section{Drug History}

Use of NSAID was noted down for only 82 participants, 9 of them were CRC patients. Using any antibiotics or NSAID reduced the risk $86 \%$ for developing CRC.

The results of multivariable analysis are presented in tables 1 to 3 . After adjustment weight loss, consumption of rice and vegetables, hematochezia and use of NSAID hold significant relationship with CRC. Among hazardous predictors the highest odds was retained by hematochezia followed by weight loss. None of the addictive behaviors prevailed significant association after controlling the effect of other predictors of CRC.

\section{Discussion}

This case control study was designed to scrutinize effect of certain factors in the development of CRC from the low-resourced population residing in a metropolitan city of Pakistan. Other than personal history, dietary practices and bowel habits, we included Asian specific addictive behaviors as putative factors for CRC.
Hematochezia followed by weight loss showed ominous association in both univariable and multivariable analyses. Smoking revealed to be another risk factor for the disease under study. Contrarily, consuming vegetables and rice exhibited protective effect heretofore.

The predisposition of $\mathrm{CRC}$ at younger age was affirmed by previous literature (Anwar et al., 2008; Amini et al., 2013). Hereditary descent led increased risk of $\mathrm{CRC}$ in young population especially from developing country (Amini et al., 2013). Our study reaffirmed the findings from literature depicting higher risk of CRC with family history of cancer. Álvarez-Cuenllas et al. suggested that patients with family history might require different strategy of screening CRC because such individuals possessed elevated chances of enduring the same disease. (Álvarez-Cuenllas et al., 2015). They also inferred older age and male gender encompassed greater risk in alignment with other studies (Torre et al., 2015). We therefore performed a matched case-control study with respect to age and gender so that effect of these two non-modifiable risk factors could be extenuated.

Although, majority of the studies enlightened high body mass index fostered hazards of CRC (Lund et al., 2011; Mehta and Shike, 2014; Aykan et al., 2015; Simons et al., 2015). In contrast, our study stipulated loss of weight jeopardized the condition imparting CRC. We envisaged the reason behind this contradicted finding that since we collected data from a tertiary care public sector hospital where patients belonged to low socio-economic status. Hence, low weight would be the intrinsic characteristics they preserved. Though, this finding is concurrent to a study from Zimbabwe which is also a low-resourced populous country (Katsidzira et al., 2015). Loss of appetite also showed ominous relationship which would contribute weight loss and hence development of CRC. Malnutrition and weight loss were also declared as ubiquitously presented among postoperative CRC patients in Brazil (Barbosa et al., 2014).

Changes in life style factors and dietary patterns deteriorate health condition (Vargas and Thompson, 2012; Mahfouz et al., 2014; Mehta and Shike, 2014). The effect of these factors elevates risk of CRC also. Western foods and high fats diets including meat imperil the conditions (Lund et al., 2011; Aykan et al., 2015). Studies extensively emphasized use of plant based diets to reduce the extent of the disease (Chen et al., 2015). The proportion of use of high fat diet was notably high in our setup and deduced positive association too. Other than indulging vegetables, consumption of rice contributed protective effect for CRC. Vargas et al. reviewed the mutation of CRC and evaluated that since vegetables and low fat diets characterized with anti-inflammatory enzymes which contributed modulation in cancer cell proliferation and apoptosis (Vargas and Thompson, 2012).

Till now, use of smoking and alcohol intake have been contemplated to be solely addictive behaviors elevating risk of CRC. We conceived to observe effect of indigenous addictive products which are consumed more by South Asian natives. Because these products empirically proven to develop other carcinoma chiefly oral and esophagus cancer (Saeed et al., 2012; Banerjee et al., 2014; Garg et 
al., 2014; Merchant and Pitiphat, 2015). Basically, these smokeless products also contain tobacco or taken with tobacco products which produce analogous impact as of cigarette smoking. From a meta-analysis of 24 cohort studies, alcohol intake enhanced risk of CRC up to $10 \%$ (Roswall and Weiderpass, 2015). This risk was 3.9 times higher among participants who used alcohol in our study. Albeit, the large likelihood would also be due to low frequency of alcohol intake in our population due to religious and cultural reasons. Gong et al. proclaimed that even after refraining cigarette smoking, the risk of CRC remained increasing (Gong et al., 2012). They found the elevated chances of 1.26 times in current smokers and 1.11 times in former smokers from a study with large sample size. Out study showed twice the risk of smoking as compare to their study. Use of paan exhibited 7 to 15 folds higher likelihood of developing oral cancer (Merchant and Pitiphat, 2015). This addictive product revealed 2 folds higher chances of developing CRC from our study. Gutka on the other hand also exhibited similar effect for the same. Therefore, cautions must be derived while using these indigenous addictive products to attenuate risk of CRC in our population.

Among bowel habits, literature demonstrated many folds higher likelihood of inflammatory bowel diseases (Jess et al., 2012a; Jess et al., 2012b). Constipation and hematochezia are also consequents of inflammatory enzymes. These two alteration in bowel habits showed significant positive association. A five year follow-up of 263 Chinese patients revealed the highest hazards due to constipation for CRC (Tang et al., 2013). While in our study, hematochezia elicited the largest frequency and risk. The implicit reason would be that since in our selected setup most of the patients visit the hospital usually at advanced stage. Hematochezia is considered secondary to $\mathrm{CRC}$ and a distinct symptom among such vulnerable individuals. Late presentation by population with low resources and financial constraints was also proclaimed in a study from Sans Francisco General Hospital (Ho et al., 2013). Other bowel habits in CRC patients were concomitant with general populations. This finding could be related to two studies on Chinese and Taiwanese CRC patients (Pan et al., 2011; Tang et al., 2013). Like Tang el. determined constipation as the highest hazardous factors out of four irritable bowel syndromes (Tang et al., 2013). Pan et al. ascertained decreased risk of diarrhea and other frequent defecation habits while ascribed these to post-operative symptoms distress among CRC patients in Taiwan (Pan et al., 2011).

Several caveats must be taken prior to interpreting findings from this study. First of all, we conducted the research on populations from low socio-economic status. Secondly, sample size of the study was limited and small even though the study elongated for a period of 5 years. Thirdly, due to small sample size some of the factors did not produce significant effect on CRC at multivariable stage whereas those factors were considered hazardous in the literature. Though, the study has also many pros which would amplify strength of the findings. Matching of the patients with their siblings, neighbors and friends elicited adjustment of many confounding factors including socio-demographic variables. Another strength was that patients admitted in our hospital came from different regions due to free availability of care, hence they might only represent the low-resourced population groups in the region. Lastly, for the first time in literature hazardous effect of indigenous addictive behaviors was investigated in this study.

In conclusion, the study acknowledged positive relationship of family history, weight loss, high fat diet, constipation and hematochezia for developing CRC. Use of vegetables and rice might assist improvement in the condition. The hazardous effects of smoking and alcohol were aligned with the literature. The ominous association of Asian specific addictive behaviors was established from our study. Further studies are needed to validate the findings produced by this research.

\section{Acknowledgements}

We would like to acknowledge students from department of Statistics who assisted us in collection of data from controls and all the participants for their kind cooperation on providing their information. We are also grateful to Dr. Kashif Shafique, Associate Professor, School of Public Health, Dow University of Health Sciences for his continual guidance at different stages of study.

\section{References}

Álvarez-Cuenllas B, Díez-Rodríguez R, Vaquero L, et al (2015). Descriptive analysis of endoscopic findings in patients with a family history of colorectal cancer. Revista de gastroenterologia de Mexico.

Amini AQ, Samo KA, Memon AS (2013). Colorectal cancer in younger population: our experience. J Pak Med Assoc, 63, 1275-7.

Anwar N, Badar F, Yusuf MA (2008). Profile of patients with colorectal cancer at a tertiary care cancer hospital in Pakistan. Annals of the New York Academy of Sciences, 1138, 199-203.

Aykan NF, Yalçın S, Turhal NS, et al (2015). Epidemiology of colorectal cancer in Turkey: A cross-sectional disease registry study (A Turkish Oncology Group trial). Turk $J$ Gastroenterol, 26, 145-53.

Banerjee SC, Ostroff JS, Bari S, et al (2014). Gutka and tambaku paan use among South asian immigrants: a focus group study. J Immigrant Minority Health, 16, 531-9.

Barbosa LRL, Lacerda-Filho A, Barbosa LCL (2014). Immediate preoperative nutritional status of patients with colorectal cancer: a warning. Arquivos de gastroenterologia, 51, 331-6.

Basharat S, Kassim S, Croucher R (2012). Availability and use of Naswar: an exploratory study. J Pub Health, 34, 60-4.

Bhurgri Y, Khan T, Kayani N, et al (2011). Incidence and current trends of colorectal malignancies in an unscreened, low risk population. Asian Pac J Cancer Prev, 12, 703-8.

Bishehsari F, Mahdavinia M, Vacca M, et al (2014). Epidemiological transition of colorectal cancer in developing countries: environmental factors, molecular pathways, and opportunities for prevention. World J Gastroenterol, 20, 6055.

Chen Z, Wang PP, Woodrow J, et al (2015). Dietary patterns and colorectal cancer: results from a Canadian population-based study. Nutr $J, \mathbf{1 4}, 8$.

Garg A, Chaturvedi P, Gupta PC (2014). A review of the systemic adverse effects of areca nut or betel nut. Ind J Med Paed 
Dietary Practices, Addictive Behavior and Bowel Habits and Risk of Early Onset Colorectal Cancer: a Case Control Study Oncol, $\mathbf{3 5}, 3$.

Gong J, Hutter C, Baron JA, et al (2012). A pooled analysis of smoking and colorectal cancer: timing of exposure and interactions with environmental factors. Cancer Epidemiol Biomarkers Prev, 21, 1974-85.

Ho C, Kornfield R, Vittinghoff E, et al (2013). Late presentation of colorectal cancer in a vulnerable population. Am J Gastroenterol, 108, 466-70.

Hooper GW, Biega T, Ginat DT (2015). Betel Nuts. In 'Neuroimaging Pharmacopoeia', Eds Springer, 79-81

Jess T, Rungoe C, Peyrin-Biroulet L (2012a). Risk of colorectal cancer in patients with ulcerative colitis: a meta-analysis of population-based cohort studies. Clin Gastroenterol Hepatol, 10, 639-45.

Jess T, Simonsen J, Jørgensen KT, et al (2012b). Decreasing risk of colorectal cancer in patients with inflammatory bowel disease over 30 years. Gastroenterology, 143, 375-81.

Katsidzira L, Gangaidzo IT, Mapingure MP, et al (2015). Retrospective study of colorectal cancer in Zimbabwe: Colonoscopic and clinical correlates. World J Gastroenterol, 21, 2374.

Khan M, Bari H, Raza S (2011a). Early postoperative outcome after curative colorectal cancer surgery. Singapore Medical Journal, 52, 195-200.

Khan MR, Bari H, Zafar SN, et al (2011b). Impact of age on outcome after colorectal cancer surgery in the elderly-a developing country perspective. BMC surgery, 11, 17.

Lund EK, Belshaw NJ, Elliott GO, et al (2011). Recent advances in understanding the role of diet and obesity in the development of colorectal cancer. Proc Nutr Society, 194-204.

Mahfouz EM, Sadek RR, Abdel-Latief WM, et al (2014). The role of dietary and lifestyle factors in the development of colorectal cancer: case control study in Minia, Egypt. Central European journal of public health, 22, 215-22.

Malekzadeh F, Sadaf GS, Poustchi H, et al (2015). Burden of gastrointestinal and liver diseases in Iran: estimates based on the global burden of disease, injuries, and risk factors study, 2010. Middle East J Digest Disease, 7, 138-54.

Malik AI, Badar F, Syed AA, et al (2015). Surgically treated rectal cancer patients-Outcomes at a tertiary care cancer hospital in Pakistan. Asian J Surg, 38, 13-20.

Mehta M, Shike M (2014). Diet and physical activity in the prevention of colorectal cancer. J National Comprehensive Cancer Network, 12, 1721-6.

Merchant AT, Pitiphat W (2015). Total, direct, and indirect effects of paan on oral cancer. Cancer Causes \& Control, 26, 487-91.

NICE (2011). Appendix 1: Summary of the 5th edition of the TNM staging system for colorectal cancer and comparison with Dukes' stage. In 'Colorectal Cancer: The Diagnosis and Management of Colorectal Cancer', Eds Cardiff,

Pan L-H, Tsai Y-F, Chen M-L, et al (2011). Symptom distress and self-care strategies of colorectal cancer patients with diarrhea up to 3 months after surgery. Cancer Nursing, 34, 1-9.

Roswall N, Weiderpass E (2015). Alcohol as a risk factor for cancer: existing evidence in a global perspective. J Prev Med Pub Health, 48, 1.

Saeed M, Muhammad N, Khan SA, et al (2012). Assessment of potential toxicity of a smokeless tobacco product (naswar) available on the Pakistani market. Tobacco control, 21, 396-401.

Simons C, Schouten L, Godschalk R, et al (2015). Body size, physical activity, genetic variants in the insulin-like growth factor pathway, and colorectal cancer risk. Carcinogenesis, bgv077.

Tang Y-R, Wang P, Yin R, et al (2013). Five-year follow- up of 263 cases of functional bowel disorder. World $J$ Gastroenterol, 19, 1466.

Torre LA, Bray F, Siegel RL, et al (2015). Global cancer statistics, 2012. CA: A Cancer J Clin, 65, 87-108.

Vargas AJ, Thompson PA (2012). Diet and nutrient factors in colorectal cancer risk. Nutrition in Clinical Practice, 27, 613-23. 